\title{
Utility of pT3 substaging in lymph node-negative urothelial carcinoma of the bladder: do pathologic parameters add to prognostic sub-stratification?
}

\author{
Moritz Maas ${ }^{1} \cdot$ Johannes Mischinger $^{2} \cdot$ Eva Compérat $^{3} \cdot$ Marcus Scharpf $^{4} \cdot$ Falko Fend $^{4} \cdot$ Tilman Todenhöfer $^{5}$. \\ Arnulf Stenzl ${ }^{1} \cdot$ Georgios Gakis ${ }^{6} \cdot$ Steffen Rausch ${ }^{1}$
}

Received: 1 November 2020 / Accepted: 8 April 2021 / Published online: 21 April 2021

(c) The Author(s) 2021

\begin{abstract}
Purpose The value of bladder cancer (BC) substaging into macroscopic (pT3b) and microscopic (pT3a) perivesical fat extension in lymph node ( $\mathrm{Ln}$ )-negative patients is controversially discussed and limited evidence for prognostic relevance of additional histopathological factors in pT3 BC exists. We evaluated the prognostic value of pT3 substaging and established pathological and clinical parameters with focus on tumor invasive front (TIF) and tumor size.

Methods Specimens of 52 patients treated with radical cystectomy (RC) for pT3 a/b muscle-invasive BC were reviewed and re-evaluated by a pathologist specialized in uropathology. Clinical variables and standard histopathologic characteristics were assessed including TIF and tumor size. Their value as prognosticators for overall survival (OS) and recurrence-free survival (RFS) was evaluated.

Results Mean age of patients was 67.55 years. Tumors were staged pT3a in 28 patients (53.8\%) and pT3b in 24 (46.8\%). Median OS was 34.51 months. Median tumor size was $3.2 \mathrm{~cm}$, median TIF was $11.0 \mathrm{~mm}$. Differences in OS between pT3a and pT3b were not significant $(p=0.45)$. Carcinoma in situ (CIS) and lymphovascular invasion (LVI) were significantly associated with pT3b tumors. Univariate analysis could not identify pathological prognosticators like TIF or tumor size for OS and RFS ( $p$ for all $>0.05$ ).

Conclusion No significant differences in OS or RFS were observed comparing Ln-negative pT3 BC following radical cystectomy. Additional pathologic variables like TIF could not be identified as prognosticator. Relevance of pT3 BC substaging needs reevaluation in larger prospective cohorts.
\end{abstract}

Keywords Muscle-invasive bladder cancer $\cdot$ Pathological staging $\cdot$ Patient outcome $\cdot$ Perivesical extension $\cdot$ Tumor invasion front

Georgios Gakis

georgios.gakis@gmail.com

1 Department of Urology, University Hospital, Tübingen, Germany

2 Department of Urology, University Hospital, Graz, Austria

3 Department of Pathology, University Hospital, Wien, Austria

4 Department of Pathology, University Hospital, Tübingen, Germany

5 Clinical Trials Unit, Studienpraxis Urologie, Nürtingen, Germany

6 Department of Urology, University Hospital Würzburg, Oberdürrbacher Straße 6, 97080 Würzburg, Germany

\section{Introduction}

Bladder cancer (BC) is one of the ten most common malignancies worldwide and the second most common uro-oncological entity [1]. Urothelial carcinoma represents the largest proportion; about $30 \%$ of the diseases are muscle-invasive at the time of their diagnosis or will become muscle-invasive in the course of their progress [2].

In patients with muscle-invasive urothelial bladder cancer (MIBC), radical cystectomy with bilateral lymphadenectomy is the treatment of choice for patients with non-metastatic disease [2]. The removed surgical specimen is pathologically examined and classified according to the American Joint Committee on Caner (AJCC) TNM staging system. This classification helps the treating physician to evaluate 
the disease, decide on necessary treatment options (e.g. need for adjuvant therapies) and to estimate the prognosis.

One important aspect of this classification is the pathologic tumor stage (pT). According to the currently applied classification, tumors extending the bladder without invading surrounding organs are separated into pT3a and pT3b. In this context, pT3a tumors show a microscopic and pT3b a macroscopic invasion of the perivesical fat. The prognostic significance of this distinction remains subject of controversial debates: In comparison to pT2 tumors, pT3 disease including both, pT3a and pT3b is associated with impaired prognosis [3]. However, this prognostic relevance has not consistently been reported for the comparison of pT3a and pT3b tumors, which led to discussion about the clinical importance of the pT3 sub-classification [4].

Among other clinicopathological variables with putative impact on the prognostic relevance are tumor size or the histopathological tumor invasion front (TIF) which was earlier discussed as a discriminative variable in pT3 bladder cancer [5].

Therefore, the aim of our study was to evaluate the prognostic value of pT3 substaging in dependence of additional histopathological variables including TIF and tumor size in patients with Ln-negative MICB.

\section{Patients and methods}

From our institutional bladder cancer database, medical records of patients with diagnosis of $\mathrm{pT} 3 \mathrm{a} / \mathrm{b}$ MIBC that underwent radical cystectomy from 2004 to 2013 were identified. Patients with other than urothelial cancer, lymph-node metastases, or unknown nodal status were excluded from the analysis.

Files and original pathology reports were reviewed for clinical and pathologic standard variables tumor category (pT) and grade (G), lymphovascular invasion (LVI), concomitant carcinoma in situ (CIS), microvascular invasion (V1), surgical margin status (R0/1). Staging and grading was performed according to the AJCC TNM system (2015 version), and the World Health Organization staging system, respectively. All pathology specimens were re-evaluated by one uropathology specialist (E.C.) and additional pathologic parameters tumor size and tumor invasion front, defined as the maximum width of the tumor invasion front in the perivesical fat, were evaluated for the final analysis. Followup of patients after surgery was performed in an outpatient setting according to the respective contemporary guideline (European Association of Urology Guidelines; S3-Guideline of the German Society of Urology) recommendations. All included patients could be followed up for tumor recurrence and death.
Chi-square tests were performed to assess the association of individual clincopathological risk-factors. For categorical analyses, tumor size and TIF were grouped according to their median values. Kaplan-Meier analyses were performed to evaluate overall survival (OS) and recurrencespecific survival (RFS). Differences between subgroups were evaluated using Log-rank test. Statistical significance was regarded as $p<0.05$. For statistic analysis, commercial software (MedCalc ${ }^{\circledR}$; Version 12.7.3.0) was used. Written informed consent was obtained by all participants. The study was approved by the Ethics Committee of the University of Tübingen (Approval Number: 417/2010 A).

\section{Results}

We identified 52 node-negative patients with $\mathrm{pT} 3 \mathrm{a} / \mathrm{b}$ urothelial cancer that underwent radical cystectomy and lymphadenectomy. Of the patients, $21.2 \%$ were female and $78.8 \%$ were male. One (1.9\%) of the patients received neo-adjuvant chemotherapy prior to radical cystectomy, two patients (3.8\%) received adjuvant platinum-based chemotherapy. The median age at surgery was 67.55 years (range 46.43-88.99). Median follow-up was 33.74 months (mean: 51.8; range 1-184.5). Of all individuals with stage pT3, 53.8\% were staged pT3a and 46.2\% pT3b. Median tumor size was $3.2 \mathrm{~cm}$ (95\% CI 2.6-3.5\% cm) and median TIF was $11.0 \mathrm{~mm}(95 \%$ CI $7.0-15.53 \mathrm{~mm}$ ). Detailed patient and tumor characteristics are summarized in Table 1.

Overall, 21 patients (40.4\%) had cancer recurrence, 33 (63.5\%) patients died during follow-up. Table 2 provides survival data for the analyzed patient cohort.

Median overall survival (OS) was 34.51 months (24.762-89.458) for the entire cohort and 60.07 months (30.443-134.483) for pT3a patients, while the detected OS for pT3b patients accounted for 33.62 (17.800-89.458) months. The observed differences in OS were however not statistically significant. In addition no correlation between pT3 substage and RFS was identified (data not shown). Association of pT3a/b substage to the individual pathologic factors revealed CIS and LVI to be significantly associated with pT3b (Table 3).

Overall, univariate analysis could not identify additional prognosticators of OS in the $\mathrm{pT} 3 \mathrm{a} / \mathrm{b}$ subgroup as shown in Fig. 1.

\section{Discussion}

The current AJCC TNM classification distinguishes bladder tumors into pT3a (microscopic perivesical tissue invasion) and pT3b (macroscopic perivesical tissue invasion) sub-stages. Taking anatomical and biological aspects into 
Table 1 Patient characteristics $(n=52)$

\begin{tabular}{|c|c|c|}
\hline Variable & $N$ & $\%$ \\
\hline \multicolumn{3}{|l|}{ Gender } \\
\hline Male & 41 & 78.8 \\
\hline Female & 11 & 21.2 \\
\hline \multicolumn{3}{|c|}{ Age (years) at surgery (Radical Cystectomy) } \\
\hline Median (range) & $67.55(46.43-88.99)$ & \\
\hline \multicolumn{3}{|l|}{ Pathological stage } \\
\hline pT3a & 28 & 53.8 \\
\hline pT3b & 24 & 46.8 \\
\hline \multicolumn{3}{|l|}{ Pathological grade } \\
\hline $\mathrm{G} 2$ & 15 & 29.4 \\
\hline$>\mathrm{G} 2$ & 36 & 70.6 \\
\hline N/A & 1 & - \\
\hline \multicolumn{3}{|c|}{ Carcinoma in situ (CIS) } \\
\hline No & 35 & 67.3 \\
\hline Yes & 17 & 32.7 \\
\hline \multicolumn{3}{|c|}{ Lymphovascular invasion (LVI) } \\
\hline No & 32 & 61.5 \\
\hline Yes & 20 & 38.5 \\
\hline \multicolumn{3}{|l|}{ Necrosis } \\
\hline No & 44 & 84.6 \\
\hline Yes & 8 & 15.4 \\
\hline \multicolumn{3}{|c|}{ Vascular invasion (V1) } \\
\hline V0 & 45 & 88.2 \\
\hline V1 & 6 & 11.8 \\
\hline N/A & 1 & - \\
\hline \multicolumn{3}{|c|}{ Surgical margin status } \\
\hline R0 & 50 & 96.2 \\
\hline $\mathrm{R} 1$ & 2 & 3.8 \\
\hline $\mathrm{R} 2$ & 0 & - \\
\hline \multicolumn{3}{|l|}{ Tumor size $(\mathrm{cm})$} \\
\hline Median & $3.2 \mathrm{~cm}(95 \%$ CI $2.6-3.5)$ & \\
\hline Arithmetic mean & 3.35 (95\% CI 2.8-3.85) & \\
\hline \multicolumn{3}{|c|}{ Tumor invasion front (TIF) in mm } \\
\hline Median & $11(95 \%$ CI 7-15) & \\
\hline Arithmetic mean & 12.7 (95\% CI 10-15.5) & \\
\hline
\end{tabular}

account, this subdivision is evident and one would expect a worse prognosis for tumors with macroscopic invasion of the surrounding perivesical tissue: The lymphatic vessel topography shows larger lymphatic vessels more distant from the bladder's serosa, resulting in a higher probability of lymphonodal spread in pT3b tumors $[6,7]$. Corresponding observations were made by Neuzillet et al. and Tilki et al. showing more frequent LN invasion in pT3b tumors than in pT3a tumors [8, 9]. Furthermore, a macroscopic invasion is usually associated with a higher tumor burden, which leads to an increased probability of phenotypic mutations for metastatic spread and a higher probability of circulating tumor cells, capable of forming metastases $[9,10]$.
Despite these theoretical considerations, the expected prognostic difference between pT3a and pT3b tumors has not been consistently observed in the literature and thus leads to a discrepancy between the existing staging classification and its prognostic implication. Particularly in patients with pT3 pN0 disease no clear conclusion regarding the prognostic significance of the sub-classification is evident:

A large multicenter study by Tilki et al. shows significant differences in cancer-specific survival (CSS) and recurrencefree survival (RFS) for 456 patients with pT3, pN0 bladder cancer [8]. These observations are supported by Sonpavde et al.: Their multicentric analysis of 578 patients shows the prognostic potential of the $\mathrm{pT} 3 \mathrm{a} / \mathrm{b}$ sub-classification for RFS and postulates a prognostic risk model for patients with pT3 
Table 2 Tabular summary of survival data for the analyzed cohort

\begin{tabular}{ll}
\hline Patients with recurrence & $n=21$ \\
\hline Location of recurrence & $n=4$ \\
Osseous & $n=2$ \\
Pulmonary & $n=5$ \\
Local recurrence & $n=2$ \\
Hepatic & $n=4$ \\
Lymphonodal recurrence & $n=2$ \\
Peritoneal carcinomatosis & $n=1$ \\
Renal recurrence & $n=1$ \\
Soft tissue & $n=33$ \\
\hline Death & $n=17$ \\
\hline Cause of death & $n=2$ \\
Death after recurrence & $n=14$ \\
postoperative complications & \\
non-cancer related deaths & \\
\end{tabular}

Table 3 Association of pathological variables with pT3 substaging

\begin{tabular}{ll}
\hline Variable & $P$ \\
\hline Grade $>2$ & 0.7508 \\
LVI & $\mathbf{0 . 0 3 2 9}$ \\
Necrosis & 0.1634 \\
CIS & $\mathbf{0 . 0 4 7 2}$ \\
Positive surgical margin & 0.5405 \\
TIF $>11$ mm & 0.2912 \\
Tumor size $>3.2 \mathrm{~cm}$ & 0.1624 \\
V1 & 0.7782 \\
\hline
\end{tabular}

LVI lymphovascular invasion, CIS carcinoma in situ, TIF tumor invasion front, V1 vascular invasion

$p$-values in bold indicate statistical significance

pN0 based on T3 substage at radical cystectomy, lymphovascular invasion and margin status [11]. Important limitations of the aforementioned studies are caused by their multicenter nature. Here, the pathology was not centrally reviewed and they cover long periods of time, possibly including different surgical and pathological approaches.

Conversely, a number of smaller, mainly monocentric studies in the mentioned subgroup pT3 pN0 revealed no prognostic impact of the pT3 sub-classification: Boudreaux et al. could not find any significant difference concerning OS, CSS and RFS between lymph node-negative pT3a and pT3b tumors ( $p=0.79 ; p=0.21$ and $p=0.53$, respectively) [12]. Similar observations were made by Dincel et al., Kim et al. and Quek et al. [13-15]. Scosyrev et al. retrospectively evaluated the SEER database (Surveillance, Epidemiology, and End results) and found impaired survival rates of pT3a $\mathrm{BC}$ as compared to $\mathrm{pT} 2 \mathrm{~b}$ disease with the same nodal status. Furthermore, they revealed an increased risk of nodal metastases with increased substaging and a decreased survival in pT3b patients compared to pT3a patients for node-positive tumors. However, in the node-negative subgroup, pT3a/b substage did not significantly impact OS [3]. Table 4 summarizes the available evidence on the prognostic impact of substaging in lymph node-negative pT3 patients.

The results of our study are consistent with the reported studies above: We could not prove any negative prognostic influence of pT3b substaging compared to the pT3a patients. Moreover, given the assumption that pT3 disease may be pathologically classified and sub-stratified by alternative pathological variables, we sought to evaluate tumor size and TIF. As for the other standard pathological factors, these parameters were not shown to be prognosticators in the present analysis.

In a comparative approach, Zarei et al. evaluated the prognostic value of the depth of tumor invasion into perivesical fat in pT3 tumors and demonstrated a significant improved CSS in patients with less than $4.5 \mathrm{~mm}$ invasion $(p=0.02)$ [5]. A combination of both parameters, width and depth of tumor invasion, could provide new insights in this context.

Our study has several limitations which have to be addressed: First, the inherent limitation of any retrospective study should be mentioned. Additionally, it has to be 

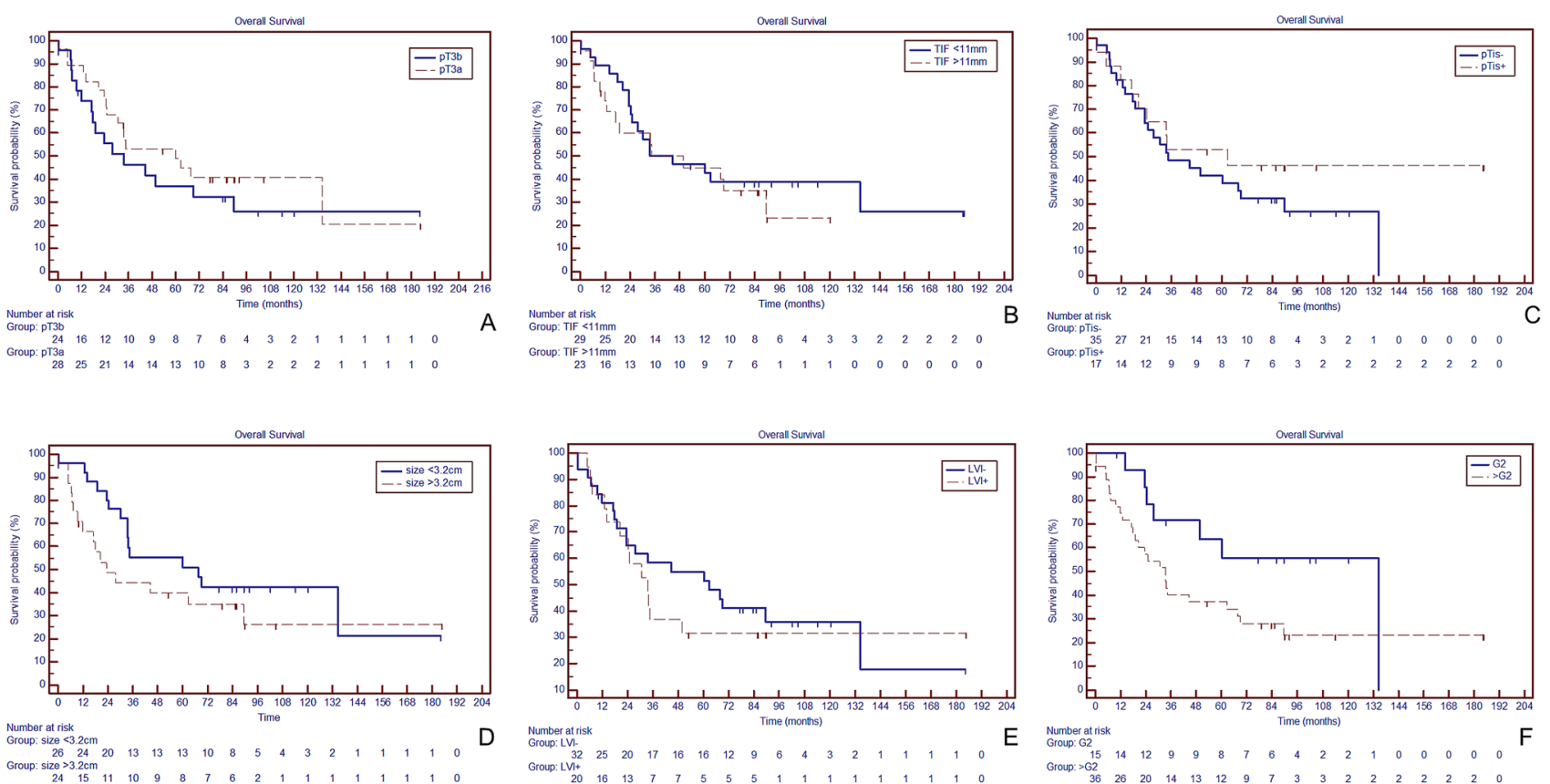

Fig. 1 Overall survival in dependence of $\mathrm{pT} 3 \mathrm{a} / \mathrm{b}$ substaging and pathological variables. (TIF tumor invasion front, $L V I$ lymphovascular invasion, $G$ grade); all $p>0.05$

noted that we reviewed a relatively small cohort of patients, which is may lead to underestimation of statistical effects. A particular strength of our evaluation is the central review of the pathological specimen by an expert in uropathology, reducing a potential inter-observer bias in the pathological assessment. Moreover, the evaluated collective is a highly selected group of advanced tumors without the prognostically significant factor of $\mathrm{pN}+$ status. This limits the comparability to other advanced tumor collectives and impairs the correlation between RFS and OS, as there is a comparatively large number of patients with long follow-up without recurrence. It results in an increased probability of non-cancer related death.

Since the estimation of the patient prognosis is a major goal of the AJCC staging system and the previous retrospectively collected data are contradictory in their statement, a prospective randomized trial with central pathological evaluation would be desirable to clarify the necessity of a sub-classification into pT3a and $b$.

\section{Conclusion}

In our study we observed no significant difference in overall survival or recurrence-free survival in the comparison of pT3a to pT3b tumors without lymph node invasion. We further could not demonstrate a prognostic value of the length of the tumor invasion front. Further prospective randomized studies are needed to assess the requirement for pathological substaging into pT3a and pT3b. 


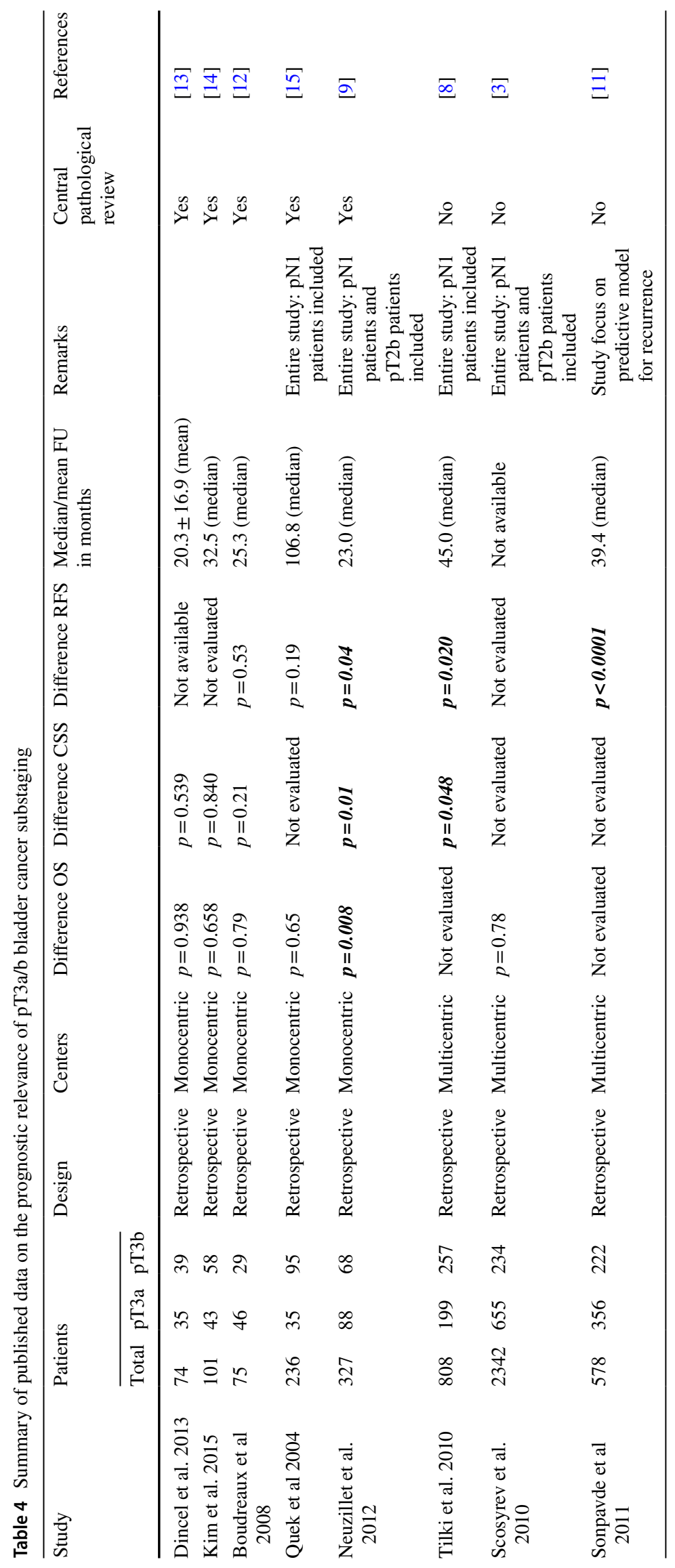


Author contributions MM: manuscript writing, editing, data collection and management; JM: data collection and management, data analysis; EC: project development, pathological examination; MS: data collection or management; FF: protocol development; TT: protocol development; AS: protocol development; GG: project and protocol development, data collection and management; SR: manuscript writing, editing, data management and analysis, project development.

Funding Open Access funding enabled and organized by Projekt DEAL. This research did not receive any specific grant from funding agencies in the public, commercial or not-for-profits sectors.

Data availability Data and material available.

\section{Declarations}

Conflict of interest/competing interests The authors have no relevant conflict of interest in the context of the work presented.

Ethics approval The study was approved by the Ethics Committee of the University of Tübingen (APPROVAL number: 417/2010 A).

Consent to participate Consent to participate was given by the patients or their legal representatives.

Open Access This article is licensed under a Creative Commons Attribution 4.0 International License, which permits use, sharing, adaptation, distribution and reproduction in any medium or format, as long as you give appropriate credit to the original author(s) and the source, provide a link to the Creative Commons licence, and indicate if changes were made. The images or other third party material in this article are included in the article's Creative Commons licence, unless indicated otherwise in a credit line to the material. If material is not included in the article's Creative Commons licence and your intended use is not permitted by statutory regulation or exceeds the permitted use, you will need to obtain permission directly from the copyright holder. To view a copy of this licence, visit http://creativecommons.org/licenses/by/4.0/.

\section{References}

1. Siegel RL, Miller KD, Jemal A (2019) Cancer statistics. CA A Cancer J Clin 69(1):7-34. https://doi.org/10.3322/caac.21551

2. Witjes JA, Bruins HM, Cathomas R, Compérat EM, Cowan NC, Gakis G, Hernández V, Linares Espinós E, Lorch A, Neuzillet Y, Rouanne M, Thalmann GN, Veskimäe E, Ribal MJ, van der Heijden AG (2020) European Association of Urology Guidelines on Muscle-invasive and Metastatic Bladder Cancer: summary of the 2020 guidelines. EurUrol. https://doi.org/10.1016/j.eururo.2020. 03.055

3. Scosyrev E, Yao J, Messing E (2010) Microscopic invasion of perivesical fat by urothelial carcinoma: implications for prognosis and pathology practice. Urology 76(4):908-913. https://doi.org/ 10.1016/j.urology.2010.02.073 (Discussion 914)

4. Paner GP, Montironi R, Amin MB (2017) Challenges in pathologic staging of bladder cancer: proposals for fresh approaches of assessing pathologic stage in light of recent studies and observations pertaining to bladder histoanatomic variances. AdvAnatPathol 24(3):113-127. https://doi.org/10.1097/pap.0000000000 000152
5. Zarei S, Frank I, Boorjian SA, Thompson RH, Kim S, Weight C, Tarrell R, Thapa P, Cheville JC (2012) Prognostic significance of measured depth of invasion of urothelial carcinoma of the bladder compared to the 2010 American Joint Committee on Cancer pT2 and pT3 classifications. J Urol 188(5):1706-1711. https://doi.org/ 10.1016/j.juro.2012.07.035

6. Matsumoto K, Soh S, Satoh T, Iwamura M, Ishikawa Y, Ishii T, Baba S (2008) Distribution of lymphatic vessel network in normal urinary bladder. Urology 72(3):706-710. https://doi.org/10. 1016/j.urology.2007.12.061

7. Marchetti C, Poggi P, Farina A, Gritti A, Scelsi L, Scelsi R (1996) Structure of the initial lymphatics of the human urinary bladder with invasive urothelial tumors. Lymphology 29(3):118-125

8. Tilki D, Svatek RS, Karakiewicz PI, Novara G, Seitz M, Sonpavde G, Gupta A, Kassouf W, Fradet Y, Ficarra V, Skinner E, Lotan Y, Sagalowsky AI, Stief CG, Reich O, Shariat SF (2010) pT3 Substaging is a prognostic indicator for lymph node negative urothelial carcinoma of the bladder. J Urol 184(2):470-474. https://doi.org/10.1016/j.juro.2010.04.007

9. Neuzillet Y, Lebret T, Molinie V, Yonneau L, Herve JM, Theodore C, Botto H (2012) Perivesical fat invasion in bladder cancer: implications for prognosis comparing pT2b, pT3a and pT3b stages and consequences for adjuvant chemotherapy indications. BJU Int 110(11):1736-1741. https://doi.org/10.1111/j.1464-410X.2012. 11104.x

10. Msaouel P, Koutsilieris M (2011) Diagnostic value of circulating tumor cell detection in bladder and urothelial cancer: systematic review and meta-analysis. BMC Cancer 11:336. https://doi.org/ 10.1186/1471-2407-11-336

11. Sonpavde G, Khan MM, Svatek RS, Lee R, Novara G, Tilki D, Lerner SP, Amiel GE, Skinner E, Karakiewicz PI, Bastian PJ, Kassouf W, Fritsche HM, Izawa JI, Scherr DS, Ficarra V, Dinney CP, Lotan Y, Fradet Y, Shariat SF (2011) Prognostic risk stratification of pathological stage T3NO bladder cancer after radical cystectomy. J Urol 185(4):1216-1221. https://doi.org/10.1016/j. juro.2010.11.082

12. Boudreaux KJ Jr, Chang SS, Lowrance WT, Rumohr JA, Barocas DA, Cookson MS, Smith JA Jr, Clark PE (2009) Comparison of American Joint Committee on Cancer pathologic stage T3a versus T3b urothelial carcinoma: analysis of patient outcomes. Cancer 115(4):770-775. https://doi.org/10.1002/cncr.24110

13. Dincel C, Kara C, Balci U, Ozer K, Ozbir S, Sefik E, Gorgel SN, Girgin C (2013) Comparison of microscopic (pT3a) and gross extravesical extension (pT3b) in pathological staging of bladder cancer: analysis of patient outcomes. Int Urol Nephrol 45(2):387393. https://doi.org/10.1007/s11255-013-0381-7

14. Kim HS, Piao S, Moon KC, Jeong CW, Kwak C, Kim HH, Ku JH (2015) Adjuvant chemotherapy correlates with improved survival after radical cystectomy in patients with $\mathrm{pT} 3 \mathrm{~b}$ (macroscopic perivesical tissue invasion) bladder cancer. J Cancer 6(8):750 758. https://doi.org/10.7150/jca.12259

15. Quek ML, Stein JP, Clark PE, Daneshmand S, Miranda G, Cai J, Groshen S, Cote RJ, Lieskovsky G, Quinn DI, Skinner DG (2004) Microscopic and gross extravesical extension in pathological staging of bladder cancer. J Urol 171(2):640-645. https://doi.org/10. 1097/01.ju.0000108664.39035.51

Publisher's Note Springer Nature remains neutral with regard to jurisdictional claims in published maps and institutional affiliations. 